\title{
MIGRACIONES PENDULARES EN LA PENÍNSULA IBÉRICA DURANTE LA ÉPOCA MODERNA. NOTAS ACERCA DEL PAPEL DE LA TRASHUMANCIA EN LA DETERMINACIÓN DEL SISTEMA MIGRATORIO DE LA ESPAÑA INTERIOR ${ }^{1}$
}

\author{
José Pablo BlanCo CARRASCO \\ Universidad de Extremadura
}

Recibido: 23/06/2012

Aceptado: 18/09/2012

\begin{abstract}
RESUMEN: Este artículo se basa en la hipótesis de que las rutas trashumantes fueron un eje central en los procesos migratorios que se vivieron en la España meridional a lo largo de los siglos modernos, en especial en las zonas de interior. Durante el siglo XVIII y los primeros compases del siglo XIX, estos movimientos migratorios van a cristalizar en corrientes definitivas modeladas por las estrategias familiares de sus protagonistas.
\end{abstract}

PALABRAS CLAVE: Movimientos migratorios, Trashumancia, Época Moderna, España.

ABSTRACT: This article is based on the hypothesis that migratory routes were a central axis in the migration processes that lived in southern Spain along the modern centuries, especially in inland regions. During the eighteenth century and the early stages of the nineteenth century, these movements will become final currents shaped by family strategies of its protagonists.

KEYWORDS: Migration, transhumance, Modern Age, Spain.

En la interpretación de los movimientos migratorios en la Península Ibérica, al menos en la que tiene la larga duración como referencia, tiene una fuerza enorme la tesis de que las regiones del sur ejercían sobre los habitantes de las montañas cantábricas una atracción mantenida secularmente al menos desde los albores del

\footnotetext{
${ }^{1}$ Este trabajo se enmarca en el proyecto de investigación HAR2010-21325-C05-04, dentro del proyecto general HAR2010-21325-C05-01, del Ministerio de Educación y Cultura. Cuenta con el respaldo científico del GEHSOMP y del proyecto de investigación IPR09A100 de la Junta de Extremadura.
} 
proceso de Reconquista, intensificándose conforme fueron adquiriéndose territorios cada vez más extensos al sur de las principales cuencas meridionales por parte de los reinos cristianos ${ }^{2}$. La época moderna observó una intensificación de esta corriente convirtiendo al sur en un territorio de promisión para muchos emigrantes vizcaínos, cántabros, leoneses o gallegos. A diferencia de sus regiones de procedencia, densamente pobladas y con limitadas opciones de medro social para ellos, el campo andaluz, las grandes villas y ciudades manchegas y extremeñas, señoras de inmensos términos concejiles y necesitadas de constantes aportes de población especializada, ofrecían evidentes posibilidades de ascenso en la escala social $^{3}$. Aunque con grandes diferencias geográficas y cronológicas, estas tierras de promisión atrajeron a sus pueblos y ciudades a un contingente constante de emigrantes septentrionales.

En el contexto de la Época Moderna, la proximidad es determinante. Los principales destinos de estos emigrantes fueron las ciudades castellanas -comunidades que contribuyeron a impulsar desde el siglo $\mathrm{XVI}^{4}-$, y, más tarde y de forma mucho más selectiva, las grandes urbes de la meseta sur, del valle del Guadalquivir y las ciudades costeras, un conjunto todavía poco conocido individualmente pero no carente de estudios significativos. Poco conocidos resultan los movimientos que estos mismos emigrantes protagonizaron en otras zonas menos atractivas económicamente, carentes de la capacidad organizativa que implicó el desarrollo de la urbanización en España y consecuentemente menos intensos desde un punto

\footnotetext{
${ }^{2}$ A diferencia de otras variables mejor conocidas, los movimientos migratorios interiores carecen de un estudio de conjunto que recoja las evidencias presentadas hasta ahora por los historiadores de la población. Este déficit es especialmente llamativo en las regiones meridionales de España, lo cual no ha favorecido la matización de esta teoría. No obstante, para una visión inicial del problema, vid. LÓPEZ LÓPEZ, R.J. (1994) «Gentes del norte peninsular en Andalucía durante la Edad Moderna. Notas sobre una corriente migratoria». En EIRAS RoEl, A. y ReY CASTElaO, O. (coord.) Migraciones internas y médium-distance en la Península Ibérica.1500-1900. Xunta de Galicia, Santiago de Compostela, pp. 467-478. También interesante al respecto Domínguez OrTIZ, A.: «La inmigración de corto y medio radio en la Andalucía Moderna: modalidades y comportamientos» en DomínGUEZ ORTIZ, A., Los extranjeros en la vida española durante los siglos XVII y otros artículos, Sevilla, Diputación de Sevilla, 1996, pp. 211-242 (Ed. Álvarez Santaló, L.C.), en especial p. 214.

3 Movimientos semejantes han sido puestos de manifiesto entre otros por REY CASTELAO, O, 1994, «Movimientos migratorios en Galicia, siglos XVI-XIX» En EIRAS RoEl A. y ReY CastelaO, O. (coord..) Migraciones interiores... op. cit. Vol. II, pp.94 y ss., LANZA GARCíA, R., 1993, La población y el crecimiento económico de Cantabria durante la Época Moderna, Universidad Autónoma de Madrid, Madrid.

${ }^{4}$ Marcos Martín, A. (1994), «Movimientos migratorios y tendencias demográficas en Castilla la Vieja y León a lo largo de la Época Moderna» en EIRAs RoEl, A. y ReY CASTELAO, O. (coords.) (1994), op. cit. El autor maneja en este trabajo de síntesis datos de Valladolid, Medina del Campo, Palencia y Zamora.
} 
de vista demográfico ${ }^{5}$. En este caso se situaron el conjunto de la Castilla meridional y las regiones sur-occidentales del reino. Sin embargo, los datos conocidos hasta ahora resultan enormemente sugerentes. Las corrientes migratorias provenientes del norte tienen en Extremadura un impacto importante, selectivo ciertamente, pero presente en un conjunto amplio de localidades de medio y gran tamaño ${ }^{6}$.

Si comparamos, pues, la capacidad de atracción del interior castellano con la periferia andaluza y las grandes urbes mediterráneas, no deja de ser evidente que territorios poco densamente poblados y con escasa actividad económica - tal es el caso de Extremadura - , eran poco atractivos para los inmigrantes de las zonas más densamente pobladas; ateniéndonos a las mecánicas de establecimiento mejor conocidas, la consolidación de redes de parentesco o paisanaje en dichos territorios debía tener menos capacidad atractiva que las que cristalizaron en la baja Andalucía o Murcia, por ejemplo, y es previsible que aparezcan con la misma intensidad en otros destinos meridionales. Con esta baja intensidad como referencia, sobresalen en todos los casos los movimientos más cercanos como escenario explicativo modal de los desplazamientos, hecho que no oculta una importante especialización de la inmigración en algunas zonas, en la mayor parte de los casos organizada en torno a las actividades ganaderas. En efecto, la práctica trashumante está presente al menos en algunos aspectos importantes del desarrollo de las poblaciones que entran en contacto, directa o indirectamente. Ello nos ayudarán a perfilar la intensidad y el sentido de estos movimientos demográficos trascendentales.

El tema que aborda este artículo se centrará en las relaciones que existieron entre la trashumancia y los movimientos migratorios que se dieron en la Península Ibérica entre finales de la Edad Media y los umbrales de la España contemporánea. Nuestra intención es plantear esta reflexión desde el punto de vista de la historia social de la población apoyándonos para ello en los resultados de la reciente historiografía sobre la estructura de los movimientos migratorios de medio recorrido en España, y en especial en Extremadura, resaltando las relaciones

${ }^{5}$ Sobre la producción histórica y su valoración en Extremadura, vid. RoDRíGUEZ CANCHO, M. (2003) «Historia demográfica e historia de Extremadura: balance historiográfico modernista, 19732001» en LÓPEZ LÓPEZ, R.J. y GONZÁLEZ LOPO, D.L. Balance de la historiografía modernista, 19732001. Xunta de Galicia, Santiago de Compostela, pp. 203-222.

${ }^{6}$ Blanco Carrasco, J.P. (1999), Demografía, familia y sociedad en la Extremadura Moderna, Cáceres, Servicio de Publicaciones de la UEx. Vid. además, Blanco Carrasco, J.P. y Santillana PÉREZ, M. (2004) «Mercado matrimonial, migraciones y movilidad social en Extremadura (siglos XVI-XVIII)» In: VII Congreso de la ADEH. Granada. [http://www.adeh.org/?q=es/biblio/author/

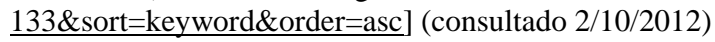


causales que se dan entre los movimientos ganaderos y los movimientos migratorios que cristalizaron en los años finales del siglo XVIII y los primeros años del XIX.

No son reflexiones diametralmente separadas. Las conexiones directas e indirectas entre población y trashumancia han sido puestas de manifiesto desde el siglo XVIII, en primer lugar, porque la práctica ganadera trashumante tiene un marcado protagonismo en los mecanismos que regulan el mercado de la tierra y ha sido el centro de numerosos estudios y reflexiones críticas, en especial como condicionante del acceso de los campesinos al principal generador de riqueza, la tierra, asegurándose en las tesis más conocidas que el promotor principal del empobrecimiento secular del interior meridional del reino de Castilla, y por tanto de su despoblación era, precisamente, la práctica trashumante organizada por el Honrado Concejo de la Mesta (Ortega López, 1986) ${ }^{7}$. Un buen resumen de esta idea es el conocido texto del padre Sarmiento

Algunos no quieren creer que la despoblación de España -decíaprocede de la Mesta, pues hay despoblados en donde no hay Mesta. Se les responde que donde no hay Mesta todo está poblado, como Galicia, Asturias, Montañas y Vizcaya \&c. La Mesta no solo despuebla la Extremadura, sino el reino de León y las Castillas por donde cruzan las cañadas que esterilizan la tierra, impidiendo que cada uno cierre su hacienda, contra todo derecho natural, romano y del país, que lo permite donde no hay Mesta ni cañadas de ella... (DOYLE, E.: Tratado sobre la cría y propagación de pastos y ganados, Madrid, 1799)

En segundo lugar, admitamos o no que la trashumancia es un agente despoblador, como es bien sabido, la despoblación selectiva debe generar diferenciales económicas entre distintos territorios, debemos asumir también que esta situación se traduce en contrastes socio-económicos que finalmente resultarán claves para explicar la intensidad y la dirección de los movimientos migratorios tradicionales, en este caso los de dirección norte-sur. Ravenstein ya postuló esta constante de la eficiencia a finales del siglo XIX y no tenemos razones empíricas para contradecirla, al menos en poblaciones históricas, a pesar de la subjetividad

${ }^{7}$ La literatura al respecto es abundante. Una de las obras más influyentes es, sin duda, el Memorial Ajustado hecho en virtud de decreto del Consejo del expediente consultivo que pende en él en fuerza de Real Orden, comunicada por la Secretaría de Estado, y de del Despacho Universal de Hacienda con fecha en San Ildefonso de 20 de julio del año de 1764. Madrid, 1771. Vid. también PEREIRA IgLESIAS, J.L. (1999) «Extremeños y trashumantes: historia de un desencuentro» En MelóN JimÉNEZ, M.A., RodríGuez GrajerA, A. y PÉREZ DíAZ, A.: Extremadura y la trashumancia. Mérida, Editora Regional de Extremadura, pp. 81-96. 
que impone en los razonamientos que explican los movimientos migratorios ${ }^{8}$. Es decir, las zonas más densamente pobladas tenderán a despejar sus poblaciones mientras que las menos densamente pobladas asumirán el papel de receptoras netas de estas expulsiones, sumando y restando entradas para compensar las deficiencias de sus respectivos balances demográficos. En este contexto se explica el constante asentamiento de vecinos de los territorios septentrionales de la corona de Castilla en sus dominios meridionales. Más allá de Ravenstein, las redes sociales establecidas incluso entre territorios muy alejados entre sí actúan como un agente vertebrador muy activo ocasionalmente. Si paulatinamente las posibilidades de mejora y medro social son percibidas de forma favorable por los pobladores de unos y otros territorios y son utilizadas por los que se encuentran en mejores condiciones de abordar este paso trascendental, encontraremos una corriente migratoria basada en determinadas constantes que se han visto facilitadas por siglos de convivencia entre los territorios septentrionales y Extremadura, en este caso concreto, a través de la práctica trashumante. En hipótesis, pues, es probable que el complejo sistema de movimientos geográficos peninsulares, organizado a partir de diferenciales económicos, ecológicos y demográficos, hiciese que las migraciones estacionales pendulares cristalizaran en asentamientos definitivos cuando las convergencias culturales, políticas y económicas entre los territorios de partida y los de llegada, unidos por paisajes compartidos secularmente, encontrasen las condiciones favorables para su cristalización.

\section{TRAZAS DEL SISTEMA MIGRATORIO DE LARGA DISTANCIA PENINSULAR.}

Los constantes flujos demográficos existentes entre el norte y el sur de España se inscriben en esta reflexión como el principal marco de referencia explicativa por cuanto su direccionalidad conectó de forma tradicional las poblaciones septentrionales del reino de castilla con La Mancha, Extremadura y Andalucía, experimentando un flujo constante que se incrementó desde finales del siglo XVIII hasta los años centrales del siglo XIX. A pesar de que los movimientos migratorios modernos se caracterizan por desarrollarse entre localidades ya intensamente conectadas, las pequeñas distancias predominantes no pueden esconder la especialización que es visible entre comarcas concretas ligadas por una tradición secular de intercambio.

\footnotetext{
${ }^{8}$ Para una revisión crítica de la obra de Ernst G. Ravenstein, ARANGo VILLA-BelDA, J.: «Las 'Leyes de las Migraciones' de E.G. Raventein cien años después» en Reis: Revista española de investigaciones sociológicas, $\mathrm{N}^{\circ} 32,1985$, págs. 7-26.
} 
En algunas comarcas cántabras, donde la emigración formaba parte estructural del modo de vida del campesino, una parte importante de la población participaba en los movimientos pendulares estacionales entre las montañas y los pueblos y ciudades de la meseta en busca de trabajo en los campos de cereal o en trabajos especializados; otros se integraban en corrientes de larga distancia con sus miras puestas en Andalucía, que era, con diferencia el destino preferido entre los emigrantes, en especial las atractivas ciudades comerciales del litoral (LANZA, 1993: 383:385). Tal ocurre con los comerciantes vascos, que se constituye en el grupo más numeroso en la Sevilla del XVIII (LÓPEZ, 1993, 382). Más al sur, la matrícula de comerciantes de Cádiz, por ejemplo, indica para las mismas fechas que el $91 \%$ de los no andaluces afincados en la ciudad procede de la periferia cantábrica ${ }^{9}$ (RUIZ, 1988, 40).

En el caso gallego, por ejemplo, los contactos entre las comarcas centrales de la actual provincia de Lugo y la Castilla meridional está perfectamente documentada a través siglos ${ }^{10}$, si bien la presencia de gallegos en el interior castellano es decreciente numéricamente conforme la época moderna llega a su fin y se abre a nuevos horizontes ${ }^{11}$. En las montañas santanderinas, la emigración hacia Andalucía y las grandes villas y ciudades manchegas y extremeñas no cesó desde la baja edad media y se transforma en migraciones estables en torno a las mismas fechas, es decir, conforme la implantación del sistema de relaciones capitalista implica mayores costes a la migración pendular y en tanto las relaciones con las recién nacidas repúblicas americanas recuperan sus relaciones con su antigua metrópolis,

${ }^{9}$ Un análisis más cercano de este período concreto pone de manifiesto que esta emigración se concentra esencialmente en las ciudades (LóPEZ, 1993, 383) y que en los lugares de destino estos emigrantes compusieron redes de colaboración que permitieron mantener un flujo constante basado en relaciones de solidaridad familiar, paisanaje o afinidad (LANZA, 1993). Es difícil saber su número concreto por las dificultades que presentan las fuentes preestadísticas pero todo apunta a que resultaron localmente importantes.

${ }^{10}$ Fernández CoRTizo, C. (1993), «'Ganando la vida con el oficio de cantero’: explotación campesina y emigración estacional en la Galicia occidental del siglo XVIII». En Eiras..., p. 337

${ }^{11}$ La independencia de las colonias americanas fue un revulsivo para la emigración de gallegos y santanderinos hacia América, quienes, junto a grandes contingentes asturianos y vascos, abandonaron en masa las tradicionales corrientes pendulares peninsulares, como los grandes campos cerealeros de la Tierra de Campos o las grandes ciudades comerciales andaluzas, en beneficio de migraciones de mayor duración en América. Hasta entonces, es Andalucía el principal territorio en el que intentar un mejorar las condiciones de vida propias y las de sus familias, normalmente residentes en los lugares de origen. La estancia, más o menos duradera, tocaba a su fin cuando se obtenían los recursos necesarios para asentarse nuevamente en su tierra natal. Vid. al respecto GARCíA LÓPEZ, J.R. (1992) Las remesas de los emigrantes españoles en América: siglos XIX y XX. Santiago de Compostela, Júcar. 
inmersa en una transformación demográfica que tiene como principal consecuencia, precisamente, la expulsión de buena parte de sus excedentes demográficos a estos destinos ${ }^{12}$.

Entre tanto, era normal, por ejemplo, que determinados sectores de la economía urbana andaluza estuvieran en manos de montañeses cántabros, hecho similar a la preferencia riojana y leonesa por asentarse definitivamente en tierras extremeñas más o menos por las mismas fechas ${ }^{13}$. En la mayor parte de los casos, estas corrientes cristalizaban en las poblaciones de destino en matrimonios que se subsumen entre corrientes estables procedentes de núcleos cercanos ${ }^{14}$. La larga cronología de intensificación de los movimientos migratorios marca los primeros años del siglo XIX como el momento en el que buena parte de las migraciones pendulares tradicionales al menos desde la baja edad media acaban por transformarse en migraciones definitivas, abriéndose una dinámica en la que el efecto llamada tiende a reforzar este movimiento a través de la participación cada vez más intensa de mujeres en el proceso ${ }^{15}$.

En la lógica emigratoria, la presencia de contactos seculares entre zonas relativamente distantes nos ayuda a relativizar la importancia la cercanía como el factor característico de la movilidad moderna. De los porcentajes conocidos se deduce que menos de un 5\% de los movimientos migratorios observados a través de las actas matrimoniales corresponden a contrayentes procedentes de localidades ajenas a las poblaciones circundantes. De hecho, la estructura zonal de las migraciones presenta la característica evidente de repartirse gradualmente en torno a un cinturón de poblaciones más o menos estable -y más menos extenso- de donde se nutre el mercado matrimonial local. No obstante, la presencia de comunidades determinadas por la especialización de sus orígenes no deja de ser significativa y sugiere que la procedencia puede ser selectiva y formar parte de la estructura general de la emigración como un elemento clave. En el caso extremeño, esta importancia creció a lo largo de la época moderna y es indispensable para comprender los movimientos migratorios que se produjeron a partir de la segunda

12 LANZA GARCía, R. (1991): Población y crecimiento económico en Cantabria durante el Antiguo Régimen. Madrid, UAM, pp. 384 y ss.

13 Blanco CARRASCO, J.P. (2012): «Trashumantes en la España moderna: de movimientos pendulares a corrientes migratorias estables» en IX Congreso de la Asociación de Demografía Histórica. Punta Delgada (Azores), 2010.

${ }^{14}$ Blanco Carrasco, J.P. (2004) «Mercado matrimonial...», op. cit.

15 Blanco Carrasco, J.P. (1999), Población, familia y sociedad en la Extremadura Moderna... op. cit. 
mitad del siglo XVIII y los primeros años del siglo XIX en el contexto de las principales villas y ciudades de la región ${ }^{16}$.

Muchos de los movimientos migratorios de larga distancia están relacionados con una dilatada tradición de contactos establecidos en función de la especialización del trabajo entre zonas relativamente alejadas, aunque no puedan ser obviados otros aspectos importantes, como las relaciones comerciales, la propiedad de la tierra y su mercado como elementos orientadores de la movilidad ${ }^{17}$. Este balance desigual entre las zonas de procedencia y destino produjo no sólo corrientes estacionales estables, sino también movimientos migratorios constantes organizados a partir de las redes establecidas en los territorios de acogida ${ }^{18}$. En Extremadura, los ejemplos no son tan llamativos y numerosos como en las ciudades andaluzas; la emigración de este tipo fue más débil, menos intensa a medida que se cerraban las posibilidades de medro social, pero los patrones son, básicamente, los mismos, en especial en torno a la actividad ganadera trashumante $^{19}$. Actividades mantenidas durante la primera mitad de la Edad

${ }^{16}$ Ibid. Ver al respecto, además, la experiencia de una serie de familias cameranas y catalanas en Cáceres en Melón Jiménez, M.A. (coord.), (1999) Antecedentes de la Cámara de Comercio de Cáceres. Cáceres, Cámara Oficial de Comercio e Industria de Cáceres. Un ejemplo significativo del Cádiz moderno en PASCUA SÁNChEZ, M.J. de la (2002) «Migraciones tradicionales: Gallegos y otras gentes del norte en Cádiz durante los Siglos XVII y XVIII» en EIRAs RoEl, A. y GonZÁlez LoPO, D. L. (coords.) Movilidad interna y migraciones intraeuropeas en la Península Ibérica, Santiago de Compostela, Publicaciones de la Universidad de Santiago de Compostela, pp. 55 y ss. De la misma autora, sobre la actividad laboral de los mandaderos gallegos «Los gallegos en el Cádiz de la Carrera de Indias. Balance secular de un proceso migratorio (1682-1778)» En EIRAS ROEL, A. y REY CASTElaO, O. (Eds.) (1994) Migraciones internas y medium-distance en la Península Ibérica, 15001900. Vol. II, Santiago de Compostela, Xunta de Galicia, pp. 845-857.

17 LANZA GARCía, R. (2002), «De norte a sur: las migraciones de la fachada cantábrica en la España Moderna». En EIRAS Roel, A. y GonZÁlez LOPO, D.L. (coords.) Movilidad interna y migraciones intraeuropeas en la Península Ibérica, Santiago de Compostela, Publicaciones de la Universidad de Santiago de Compostela, p. 19.

${ }^{18}$ Para el caso gallego, sobre el que existen numerosas referencias útiles, GonZÁLEZ LoPO, D.L. (1994) «Campesinos en movimiento. Pluriactividad, ajuste familiar y desplazamientos de los campesinos del norte de España. Siglos XVIII y XIX» En Eiras Roel, A. y Rey Castelao, O. (1994) op. cit., pp. 491-514.

19 En un primer momento, la presencia de trashumantes establecidos definitivamente es muy pequeña, casi residual, como atestiguan los estudios basados en el escrutinio de las actas matrimoniales de algunas ciudades extremeñas (vid. p.e., RodRíGUEZ SÁNCHEZ, A. (1977) Cáceres. Población y comportamientos demográficos. Cáceres. Universidad de Extremadura, pp. 200-203. Desde el punto de vista de la actividad trashumante en el mismo territorio de referencia y sobre la base de fuentes notariales y municipales, Pereira IGLESIAS, J.L. (1990), Cáceres y su tierra en el siglo XVI. Economía y Sociedad. Cáceres, Institución Cultural El Brocense). Los asentamientos de pobladores asturgalaicos y montañeses en las poblaciones extremeñas de la Trasierra están 
moderna, cristalizarán en asentamiento definitivos a lo largo del siglo XVIII. En Medina de las Torres, una pequeña población de la provincia de Badajoz, uno de los propietarios que más amplía sus bienes en los primeros años de la segunda mitad del siglo XVIII es un camerano, Joaquín López Montenegro ${ }^{20}$, vecino de Villoslada de Cameros y propietario de un nutrido conjunto de tierras, bienes inmuebles y algunas cabezas de ganado. En el mismo caso se encuentran algunos trashumantes de Valencia del Ventoso oriundos de Viniegra de Abajo ${ }^{21}$, u otros del mismo origen convertidos en propietarios en Montánchez, donde la familia Peñalosa, por ejemplo, residente en Soria, posee tanto hierbas como viñas, tierras de labor y varias casas. En Villagarcía de la Torre nos encontramos con el mismo caso, esta vez en la persona de un mayoral avecindado en Lumbreras llamado Manuel Rubio Aceñas ${ }^{22}$. Durante la segunda mitad del siglo XVIII la estructura económica de base local de los ganaderos trashumantes está comenzando a arraigar en los pastos de invernadero. El monasterio de Guadalupe, por ejemplo, que es poseedor de una enorme cabaña trashumante, presenta rasgos de especialización entre sus trabajadores tanto por la complejidad de sus actividades como por el selectivo origen de sus trabajadores foráneos. Un ejemplo de la especialización geográfica que es visible en estos casos es constatable a simple vista a través del CUADRO I, que recoge el origen geográfico de los pastores trashumantes de la cabaña merina del Monasterio de Guadalupe a mediados del siglo XVIII.

En su mayoría los pastores proceden de concejos muy determinados, agrupados en un radio relativamente estrecho del norte de León. En este caso el monasterio buscó personal procedente de los pastos de agostadero, de los concejos de Villamanín -este en concreto aporta el mayor contingente-, Sena de Luna, Barrios de Luna y Carrocera ${ }^{23}$. Es evidente que son comarcas todas ellas situadas en los tramos más septentrionales de las cañadas de la Plata, Occidental y Oriental

documentados desde el siglo XIII (Bernal EstéveZ, A. (1998) Poblamiento, transformación y organización social del espacio extremeño (s. XIII al XV) Mérida, Editoria Regional de Extremadura, pp. 259 y ss.). A lo largo de la época moderna, su presencia disminuye hasta su reactivación en el XIX. (Blanco Carrasco, J.P. (1999) Población, familia y sociedad en la Extremadura Moderna. Cáceres, Servicio de Publcaciones de la UEx.), sin embargo fue determinante hasta finales del siglo XVI.

${ }^{20}$ Archivo General de Simancas (AGS), Dirección General de Rentas (DGR) $1^{\text {a }}$ Remesa, leg. 864.

${ }^{21} \mathrm{Ibid}$, leg. 863.

${ }^{22}$ Ibid. leg, 870.

23 Estas procedencias coinciden con las trazadas por Perez ÁlVAREZ, M.J. (1996) La montaña noroccidental leonesa en la Edad Moderna. Secretariado de Publicaciones, Universidad de León. León, pp. 83-86. 
leonesa, y coincidentes en buena parte de su recorrido original con los trazados leoneses del camino de Santiago, es decir, un territorio perfectamente definido por la actividad trashumante y paso natural entre los territorios occidentales de norte a sur. Si admitimos que la información sobre la realidad económica de los zonas de acogida es trascendental para determinar la elección de los emigrantes, este conjunto de relaciones ligadas a la trashumancia son la base de un conocimiento imprescindible para propiciar movimientos migratorios entre ambas zonas, suponiendo en parte la salida natural para la sobrepoblación de las áreas más densamente pobladas. En el caso de la ciudad de Badajoz, por ejemplo, buena parte de los contrayentes foráneos registrados por los párrocos en el momento de su matrimonio proceden de los obispado de Astorga, Oviedo y León, una característica que se intensifica y hace más compleja conforme el siglo XVIII va tocando a su fin ${ }^{24}$.

Mapa I. Procedencias geográficas de los rabadanes empleados por el monasterio de Guadalupe. 1752

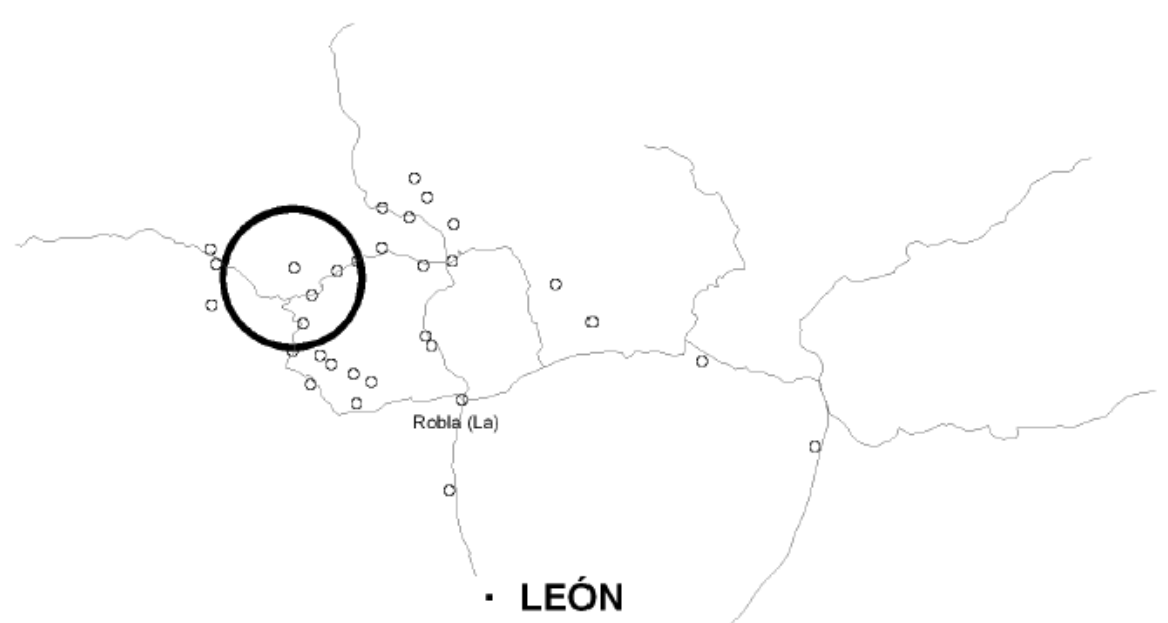

FUENTE: Vid. CUADRO I.

${ }^{24}$ Con la aparición de matrimonios en los que los dos contrayentes proceden de la misma zona geográfica, por ejemplo. BlANCO CARRASCO, J.P. (2003) «Notas para un estudio sobre las migraciones y la movilidad geográfica en el entorno urbano extremeño (1500-1860)», Revista de Demografía Histórica. 2003, XXI(1), 77-112. 
Cuadro I. Procedencia geográfica de los rabadanes al servicio del Real Monasterio de Guadalupe (1752). Concejos.

\begin{tabular}{lc}
\hline Barrios de Luna, Los & 14 \\
Boñar & 1 \\
Cármenes & 1 \\
Carrocera & 13 \\
Cistierna & 1 \\
Cuadros & 1 \\
Pola de Gordón, La & 5 \\
San Emiliano & 7 \\
Sena de Luna & 18 \\
Tonín de Arbás & 19 \\
Villamanín & 65 \\
Valdepiélagos & 2 \\
\hline FuENTE: LLOPIS AGELÁn (1990) Guadalupe. 1752. Según las \\
Respuestas Generales del Catastro de la Ensenada, Madrid, \\
Tabapress, y elaboración propia.
\end{tabular}

\section{EL SISTEMA MIGRATORIO EN EXTREMADURA. MOVIMIENTOS DE LARGA DISTANCIA.}

En hipótesis, la regularidad del sistema migratorio impone que las zonas más densamente pobladas deben comportarse como zonas de emigración neta, frente a las provincias en las que el acceso a la propiedad es más flexible, que bajo este mismo principio regulador, habrán de ser territorios de acogida. Dado que las posibilidades de asentamiento en las regiones del interior estuvieron limitadas -si no imposibilitadas- por el férreo control de la propiedad y la estrecha diversificación de la estructura del trabajo, desde el siglo XVII hasta la segunda mitad del siglo XVIII los movimientos migratorios entre las regiones cantábricas y el sur interior debieron ser sobre todo estacionales, momento a partir del cual los destinos de interior se vieron debilitados por el enorme empuje de la urbanización costera cantábrica y los destinos americanos ${ }^{25}$.

Para que cristalizasen en corrientes migratorias estables, el principal sustrato de dichos movimientos pudo ser el conocimiento de la realidad social y económica que manejaban y transmitían los pastores y ganaderos trashumantes en sus lugares de destino, un concepto difícil de medir pero plausible dentro del esquema de transmisión de información tradicional. Los territorios al norte y al sur del Tajo se beneficiaron de una secular convivencia y culturas comunes precisamente

${ }^{25}$ LANZA GARCía, R.: (2002), «De norte a sur...», op. cit. p. 48. 
alrededor de esta práctica, pues las cañadas no solo unen términos complementarios desde el punto de vista ecológico, sino que también conectan territorios con muy distintos niveles de densidad y ocupación del espacio desde el punto de vista demográfico, atendiendo a la flexibilidad del trabajo o al más etéreo concepto de la elasticidad social. Se dirá, con razón, que dichos contactos se venían dando con regularidad al menos a partir de la consolidación del proceso repoblador que acompañó a la conquistas cristianas de los reinos moros del $\operatorname{sur}^{26}$, pero es impensable que se mantuviesen de no haber persistido un hilo que uniese ambas partes del reino. Este nicho cultural común y siglos de contacto provocarían que con el paso del tiempo, los característicos movimientos estacionales acabaran cristalizando en asentamientos definitivos facilitados precisamente por ese conocimiento secular. Históricamente, pues, la explicación debe buscarse en el mantenimiento de movimientos migratorios residuales, potenciados en el momento en el que las condiciones sociales, políticas y económicas de las zonas de origen impeliesen a los vecinos a instalarse en tierras del sur.

Los contactos entre los extremos septentrional y meridional del reino de Castilla, y los que se dieron con el resto de los reinos de la monarquía en esta misma dirección, tienen una explicación sólo visible a través de las prácticas ganaderas, pero no es menos cierto que las sucesivas crisis por las que atravesó el sector, el diferencial entre las tasas de crecimiento demográfico de unas zonas y otras y los inconvenientes cotidianos que la realidad imponía ante la posibilidad de embarcarse en un proceso vital de la envergadura de un traslado definitivo, impide observar la conexión entre estos dos elementos de la dinámica social, es decir, entre la ganadería trashumante y los movimientos migratorios, como un proceso unívoco.

Es posible, pues, que las corrientes migratorias de emigrantes localizados en las zonas de procedencia de los ganados se modulasen precisamente en función de la

${ }^{26}$ Domínguez ORTiZ, A. (1996), «La inmigración de corto radio...», op. cit, pp. 215-216. LóPEZ LÓPEZ, R.J. (1994), «Gentes del norte peninsular...», op. cit. p. 377. La bibliografía modernista dedicada a la trashumancia que ha recalado en las relaciones culturales visibles en esta práctica es abundante. Desde un punto de vista general, por ejemplo, GARCIA MARTín, P. (1990), El patrimonio cultural de las Cañadas Reales. Valladolid, Consejería de Cultura de la Junta de Castilla y León. Para una interesante comprensión de las prácticas trashumantes desde el punto de vista de las cuadrillas de pastores, el original libro de Rio, M. del (1828) Vida Pastoril, Madrid, imprenta Repullés, comentado y editado por el mismo García Martín (Madrid, Ed. del Museo Universal, 1985). Para una comprensión del impacto social de la trashumancia en la Extremadura del Ochocientos vid. SÁNCHEZ Marroyo, F. (1999) «La Trashumancia en el siglo XIX. Práctica económica y mecanismo de dinaización social». En MElón JimÉnez, M.A., Rodríguez GrAJERA, A. y PÉREZ DíAZ, A. (coords.) Extremadura y la trashumancia.., op. cit. p. 109. 
evolución de largo recorrido que siguió la actividad ganadera ligada a la práctica trashumante. A finales del siglo XVI, por ejemplo, cuando la presión sobre los recursos disponibles parecía haber acorralado definitivamente a las cabañas trashumantes y el ansia de tierras era un clamor en toda Castilla, una profunda crisis en las arcas de la monarquía y el empeoramiento constante de las condiciones de vida de los pecheros, supuso un renacer de la actividad al amparo de la continua necesidad de capitales con la que convivían cotidianamente los núcleos del sur castellano, enteramente señorializados y a merced de las apetecibles rentas mesteñas. En este momento los flujos migratorios que animaron durante decenios a un conjunto no desdeñable de gallegos, riojanos, leoneses y asturianos a asentarse definitivamente en Extremadura y La Mancha -continuando un proceso repoblador iniciado en el siglo XIII-, esperanzados en obtener una parte de los extensos territorios puestos en cultivo todavía a finales del reinado de Felipe II, se desvanecieron por la quiebra de la economía campesina, comenzando un lento proceso de recuperación que en la zona meridional castellana va a presentar signos de incapacidad muy conocidos por la historiografía que perduran hasta bien entrado el siglo XVIII.

Este lentísimo proceso de recuperación determinó también que los asentamientos de emigrantes procedentes del norte densamente poblado fuese más intenso conforme las redes de acogida se fueron reconstruyendo, tal y como hemos sugerido, después que a finales del siglo XVI las regiones situadas entre el sur del Tajo y Andalucía entrasen en una profunda depresión demográfica. En definitiva, las conexiones tradicionales entre el norte y el sur regional se reactivan aproximadamente a mediados del siglo XVIII, como sugieren el caso de Plasencia, Cáceres y sus pueblos comarcanos en el caso extremeño, otras zonas del interior castellano, como las tierras de Alcaraz, y algunas ciudades del entorno andaluz en otros escenarios meridionales ${ }^{27}$. En todos estos ejemplos aparecen nítidamente dibujados movimientos de población procedentes de los agostaderos tradicionales de las cabañas trashumantes como un contingente relevante dentro del conjunto de los emigrantes conocidos.

Una evidencia gráfica me asistirá a la hora de presentar esta idea de una manera más clara. El gráfico siguiente representa la evolución de dos territorios situados en una y otra mitades del reino de Castilla. Tienen la particularidad de haber sido,

27 Blanco Carrasco, J.P. (1999) Población..., op. cit.; García GonZÁlez, F. (2000) Las estrategias de la diferencia: familia y reproducción social en la Sierra de Alcaraz. Siglo XVIII. Madrid, Ministerio de Agricultura, Pesca y Alimentación; PASCUA SÁncheZ, M.J. de la (1994) «Gallegos...», op. cit. 
durante siglos, comarcas conectadas por actividades ganaderas trashumantes, una en cada erxtremo de la Cañada Soriana. Se trata, como reza el título, de la evolución del número de nacidos en la ciudad de Cáceres y en algunas localidades de la riojana sierra de Cameros.

Lo que primero llama la atención del gráfico es la nítida división del comportamiento de la población que se produce tras el comienzo de la segunda mitad del siglo XVIII, en muchos aspectos relacionado con la crisis agraria que permitió a los agostaderos mantener un mínimo crecimiento demográfico incluso en los momentos de crisis más agudos padecidos por los invernaderos. Durante el siglo XVII la comarca de Cameros vivió una situación de impás, de espera, con un saldo final relativamente pobre pero positivo. En Cáceres, sin embargo, el saldo experimentado es muy negativo, de pérdida constante hasta el final del Seiscientos. Esta situación comienza a cambiar lentamente durante la primera mitad del XVIII, surgiendo una brecha en el comportamiento demográfico que sugiere una divergente evolución desde finales del reinado de Fernando VI y durante el reinado de Carlos III. En estas fechas, siguiendo la lógica migratoria que sugiere que la atracción de los lugares de destino tienen más impacto en territorios que comparten tradicionales lazos de dependencia - de hecho, en su formulación más sencilla esta ley asegura que no se emigra a lugares desconocidos-, los movimientos migratorios desde la zona de agostadero deben intensificarse y es ahora cuando el historiador puede encontrar referencias más abundantes de avecindamientos, matrimonios o nuevas residencias. La crisis de la actividad trashumante, en 1820, no hizo sino acrecentar esta corriente sobre la base de grupos ya establecidos que potenciaron el conocido "efecto llamada" de los trashumantes convertidos en comerciantes, financieros y ricos hombres de negocios en Extremadura.

Para comprender mejor este proceso es necesario contextualizarlo convenientemente. A lo largo de estos años, nuestras investigaciones han demostrado que el desarrollo de las ciudades y grandes núcleos extremeños posterior a la primera mitad del siglo XVIII se cimentó no tanto en un diferencial positivo de las tasas de crecimiento natural -es decir, por la diferencia creciente entre el número de nacimientos originados en el seno de la comunidad frente a un número decreciente de defunciones-, sino por la incorporación constante de emigrantes procedentes tanto de las comarcas vecinas como de territorios más distantes. Es decir, una parte esencial de su desarrollo demográfico a finales del Antiguo Régimen y las primeras décadas del siglo XIX está íntimamente ligado a la movilidad geográfica.

En Cáceres en concreto, el proceso tiene unas características que lo hacen especialmente llamativo y es un referente indispensable para conocer la realidad 
por la que pasaron buena parte de las medianas y grandes poblaciones españolas meridionales. Por una parte, está claro que el auge económico que sobrevino tras la paralización de la contienda sucesoria entre 1709 y 1715 -después de un desastroso inicio de siglo-, transformó el escenario económico regional, favoreciendo donde fue posible la puesta en cultivo de nuevos terrazgos. La forma en la que esto afectó al partido de Cáceres en su conjunto, y a las grandes poblaciones vecinas -como Brozas, por ejemplo, magistralmente estudiada por Felicísimo García Barriga ${ }^{28}$ demuestra que muy pronto los márgenes de crecimiento natural se vieron superados por el dinamismo agrario, siendo necesario un acopio continuo de población de sus respectivos marcos demográficos de influencia. No obstante, mientras pudieron, las poblaciones redujeron incluso sus contactos con otras poblaciones debido a que las estrategias familiares suelen hacer prevalecer un principio de continuidad frente a la introducción de nuevos integrantes procedentes de otros lugares distintos al de residencia. En definitiva, llegados a la mitad del siglo de las luces, la mayoría de estos núcleos moderaron las tasas de crecimiento obtenidas en los años veinte del Setecientos,.

En los lugares en donde las actividades políticas y económicas tuvieron mayor diversificación, como ocurre con la futura capital provincial, la llegada de emigrantes procedente de los pueblos comarcano no hizo más que crecer, y lo hizo nutriéndose esencialmente de jóvenes procedentes de las grandes poblaciones vecinas. Arroyo de la Luz, Malpartida de Cáceres o el Casar; Brozas, Garrovillas e incluso Alcántara van a proporcionar un número creciente de jóvenes a la capital cacereña $^{29}$, atraídos por las posibilidades de trabajo que ofrecían las nuevas dependencias gubernamentales -acuartelamientos fijos, la Real Audiencia de Extremadura $^{30}$ y la Diputación Provincial más tarde-, unas instalaciones que estaban siendo aprovechadas también por una emigración más especializada, ligada directa o indirectamente a este proceso histórico, llamada a convertirse en un nuevo reclamo emigratorio: los ganaderos mesteños. Como consecuencia de todo ello, el número de habitantes de la ciudad experimentó un crecimiento desconocido en

${ }^{28}$ GARCÍA BARRIGA, F. (2002) «Análisis de los movimientos migratorios en Extremadura a través de los libros de matrimonio: el caso de Brozas (Cáceres) durante la Edad Moderna», Actas de los XXX Coloquios Históricos de Extremadura, Trujillo, 203-225.

29 Blanco Carrasco, J.P. (1999) Población..., op. cit. Garcia Barriga, F. (2009) Familia y sociedad en la Extremadura de los tiempos Modernos. Cáceres, Universidad de Extremadura.

${ }^{30}$ La Real Audiencia de Extremadura comienza su andadura en 1790. Sobre su impacto urbano, CAMPESINo FernáNDEZ, A.J. (1982) Estruntura y paisaje urbano de Cáceres. Cáceres, Colegio de Arquitectos de Extremadura. Para una detallada crónica sobre su establecimiento, PereIRA IGLESIAS, J.L. y Melón JimÉneZ, M.A. (1991) La Real Audiencia de Extremadura. Fundación y establecimiento material. Mérida. Asamblea de Extremadura. 
toda su historia, con cifras de inmigración neta que suponen que de cada 100 habitantes ganados en este proceso, una tercera parte aproximadamente procedía de la emigración.

Gráfico 1. Evolución de los nacimientos en Cáceres y algunas localidades cameranas. Índices.



FuENTE: En el caso del Cáceres del Quinientos, RodRíGuez SÁnCHEZ, A. (1977) Cáceres... op. cit. El trabajo de Rodríguez CANCHO, M. (1981) La Villa de Cáceres en el siglo XVIII. Demografía y Sociedad. Cáceres, Caja de Extremadura, es la referencia de los datos del Setecientos. El resto, Archivo Diocesano de Coria-Cáceres, libros parroquiales de casados de San Mateo, San Juan, Santa María y Santiago. La serie camerana, GuRRía GARCíA, P.A. (2004) La población de la Rioja durante el Antiguo Régimen demográfico. Logroño, Universidad de la Rioja.

Las procedencias observadas a lo largo de los tres siglos modernos nos aportan una información de singular importancia para el tema que nos ocupa ahora, tal vez mayor incluso que la consideración cuantitativa del fenómeno. Tradicionalmente, el conjunto de las migraciones observadas en Cáceres incluía dos elementos vinculados con el espacio geográfico de referencia que la caracterizaban. Uno de ellos, ya mencionado, situaba el origen de estos emigrantes en el entorno más cercano a la villa, el partido y las grandes villas situadas entre los límites del término y la frontera con Portugal, en tierras de la Orden de Alcántara, hecho que no deja de ser del todo habitual en Extremadura y por extensión en el interior castellano exceptuando Madrid. El segundo apunta que el resto de las procedencias mantiene a largo plazo una clara orientación vertical casi paralela al meridiano, en detrimento de las poblaciones orientales, con las que apenas si se mantienen contactos. La emigración, siguió, por tanto, un sentido enteramente dependiente de las vías de comunicación, aunque, como demuestra el mapa, no tanto la compuesta 
por los caminos de herradura como por las cañadas. Por el contrario, en Badajoz, cuya estructura urbana es netamente distinta a la cacereña, los movimientos de sus emigrantes meridionales y las procedentes del interior castellano manchego tienen una relevancia creciente desde el siglo XVII ${ }^{31}$.

Conviene recordar que Cáceres estaba conectado con los cuatro puntos cardinales por una red de caminos reales precaria pero no inexistente, de manera que la estructura circular de sus migraciones de corto radio queda reflejada de forma coherente con la red caminera del partido y las conexiones con las grandes poblaciones vecinas. Sin embargo, la emigración de mayor alcance geográfico no, en parte porque se diluía en la red caminera ordenada en torno a la Vía de la Plata y el ramal que finalmente conectaba con el camino real de Madrid, y en parte por la inconsistencia de las migraciones de procedencia no septentrional puesto que las poblaciones de origen situadas por debajo del valle del Guadiana y al este de la tierra de Trujillo son prácticamente inexistentes.

Existe por tanto una dualidad que caracteriza la emigración moderna de procedencia más lejana hacia tierras cacereñas: tienen una clara fijación en el norte de la península y, en segundo lugar, su distribución parece organizarse a través de los ejes de comunicación que significan las cañadas.

Dos incógnitas surgen inmediatamente ante esta hipótesis. ¿Porqué las cañadas, vías pecuarias, adquieren un papel que está o debe estar reservado a otro tipo de conexiones? Por otra parte, ¿limitó o modificó este modelo distributivo su estructura una vez disminuida la actividad trashumante?

Aunque las cañadas no son caminos en sentido estricto ${ }^{32}$, y más de un investigador ha sugerido que se trata de rutas seguidas por los propios animales en sus desplazamientos estacionales, la coincidencia de trazados en muchos tramos entre la red caminera y el entramado de cañadas, cordeles y veredas hace irrelevante la diferenciación, y más aún en el occidente peninsular, un territorio en el que la comunicaciones tuvieron y tienen una marcada orientación norte-sur, con un desarrollo francamente deficiente de sus red de caminos, poco eficientes y mal acondicionados. Esto hacía que uno y otro trazado se superpusiesen, frecuentemente sobre una base común, romana y tardorromana.

Por tanto, aunque parezca una obviedad, conviene recordar que para emigrar se necesita una vía de comunicación que conecte un lugar con otro, bien sea

\footnotetext{
31 Blanco Carrasco, J.P. (2003) «Notas para un estudio ...», Op. cit.

32 García Martin, P. (1991) Cañadas, cordeles y veredas. Valladolid, Junta de Castilla y León.
} 
directamente, bien a través de pasos intermedios. El viajero puede internarse por bosques inhóspitos o montes escarpados, pero el emigrante sigue frecuentemente una ruta preestablecida, conocida y segura, y no son pocas las que conectan el norte con el sur peninsular, y viceversa, sobre todo en el reino castellano, un territorio modelado por estrategias de expansión que persiguen colmatar sus necesidades precisamente sobre la base de esta complementariedad.

Como es bien sabido, en el siglo XVI (1543-1546), el licenciado Villuga publicó un repertorio que dibujaba los itinerarios de los principales caminos españoles. Tiene la virtud de haber sido uno de los primeros, y fue imitado y corregido por ediciones posteriores a cargo de diferentes autores, que básicamente repitieron buena parte de sus limitaciones, como el hecho de carecer de información sobre extensas porciones de territorio del interior peninsular, en especial en Extremadura y el occidente no costero de Andalucía ${ }^{33}$. No obstante, me parece significativo traerlo ahora a la memoria por una razón: el trazado de las principales vías longitudinales tienen una correlación muy estrecha con la estructura viaria de las rutas ganaderas. El profesor Ángel Cabo describe el surgimiento de estas vías de comunicación como un proceso histórico en el que las prácticas ganaderas desarrolladas en época prerromana se constituye en el principal factor constructivo ${ }^{34}$. Las calzadas romanas se superponen a caminos determinados por el pastoreo trashumante precedente, y sobre aquellas se construye la red viaria posterior, compuesta en su parte más visible por los Caminos reales y por las carreteras más tarde ${ }^{35}$, de suerte que los trazados compartidos, los pasos comunes y las limitaciones a las que expone un territorio como el hispano determinan a ambas redes.

Aún asumiendo, de nuevo, que las cañadas no son caminos stricto sensu en ambos casos, la distancia recorrida entre estos territorios complementarios, los pasos entre las tierras altas y los grandes valles fluviales meridionales están fuertemente marcados por el clima y la orografía del terreno y es improbable que las diferencias del uso determinaran rutas alternativas. Además, los grandes nudos de comunicación del reino coincidían frecuentemente como organizadores de esta compleja red mixta, tanto dentro como fuera del reino de Castilla. Los casos de

33 Villuga, P.J. (1546) Repertorio de todos los caminos de España (hasta agora nunca visto). Madrid. (Ed. Menéndez-Pidal Goyri, 1951)

34 Cabo Alonso Á. (1991). «La Cañada Leonesa Occidental». En García Martín, P., Cañadas, Cordeles y Veredas. Op. cit.

35 Roselló í Verger, V.M., y Marías Martínez, D. (2004), Historia, clima y paisaje : estudios geográficos en memoria del profesor Antonio López Gómez. Valencia, Universidad de Valencia, 2004. pp. 105 y ss. 
Valladolid, Palencia, Medina del Campo o Villacastín, Trujillo, Cuenca, Mota del Cuervo, o Braga en el reino de Portugal pueden ser algunos de una lista realmente nutrida.

Si prestamos atención exclusivamente a las vías que atraviesan Extremadura ${ }^{36}$, se percibe con claridad que los recorridos que unen la cuenca del Guadiana con los puertos septentrionales -Tornavas, Perosín, Béjar, Puerto del Pico...- coinciden con los puertos utilizados por las cañadas, paradas frecuentes entre los caminantes y lugar de cobro a los mayorales por derechos de portazgo. El eje central que significa el itinerario que conecta Sevilla y Astorga por Extremadura era seguido por reses provenientes del obispado de Astorga y la Sierra de la Demanda y Cameros a partir de Plasencia, cuyos destinos podían ser los pastos del condado de Niebla o las tierras concejiles de Sevilla, por ejemplo. En sentido oeste, la conocida Vía da Estrela, que se prolonga en tierras extremeñas atravesando el puente Alcántara hacia Plasencia, durante buena parte del recorrido portugués enlaza con el camino utilizado por los peregrinos que viajan al santuario de Guadalupe, una vía de peregrinación cuya trascendencia en el siglo XV y XVI competía en importancia con el camino de Santiago; desde dicho monasterio, uno de los mayores propietarios de merinas del reino castellano, salían regularmente rebaños hacia las tierras de agostadero en León, para lo cual utilizaban buena parte del trazado del camino real a Madrid antes de enlazar con las cañadas occidentales en Mombeltrán. Estos itinerarios son esenciales para comprender en profundidad la naturaleza de una parte singular de los movimientos migratorios de larga distancia del interior.

Cuadro III. Concejos leoneses de procedencia de los inmigrantes cacereños. 1750-1850. San Juan de los Borregueros.

\begin{tabular}{lcc}
\hline & $1750-1799$ & $1800-1850$ \\
\hline Boñar & 1 & 2 \\
Cabrillanes & 1 & 1 \\
Castrillo de Valduerna & 1 & \\
Destriana & 1 & 1 \\
Folgoso de la Ribera & & 1 \\
Gradefes & & 1 \\
Izagre & 4 & 1 \\
Murias de Paredes & 1 & \\
Paramo del Sil & &
\end{tabular}

36 Mangas Navas, J.M. (1999), «La red nacional de vías pecuarias en el territorio de Extremadura: estrategias y diseños», En MElón JiMÉneZ, M.A., RodríGuEZ GRAJERA, A. y PÉREZ DïAZ, A. Extremadura y la trashumancia ... op. cit. pp. 143-148. 
Puebla de Lillo

Reyero

Riaño

Riello

San Emiliano

Santa María de la Isla

Valdelugueros

Valderas

Villablino

Villazala

Total

Total general
1

\begin{tabular}{ll} 
& 1 \\
1 & 1 \\
& 1 \\
& 1 \\
1 & 2 \\
& \\
& 2 \\
12 & 1 \\
\hline
\end{tabular}

29

El cuadro anterior nos ayudará a reforzar esta idea. Tal como ocurría con los rabadanes empleados en el cuidado de los rebaños del monasterio de Guadalupe, la procedencia de los leoneses asentados en la colación de San Juan en la villa de Cáceres ${ }^{37}$ está directamente relacionada con los mismos agostaderos, un corolario de concejos que se sitúa en el norte de la actual provincia de León. Más al sur, en torno a las cañadas, siguiendo su trazado, se organizan los emigrantes zamoranos desde Puebla de Sanabira a Benavente-, tal como ocurre con los palentinos, en especial en torno al trazado de la Cañada real leonesa y la Cañada de San Roque confluentes en esta latitud, ambas cercanas al curso del río Carrión. Más hacia el sur las procedencias son más difusas en tanto que el protagonismo de las cañadas se pierde en beneficio de cierta dispersión territorial. Los casos salmantinos son una buena base para sostener este argumento -las procedencias en San Juan pueden apreciarse en los MAPAS 3A. a 3C-, pero es el caso de Ávila y la franja occidental de la provincia de Toledo también, que concentran conjuntamente un $10 \%$ del total de emigrantes varones contabilizados en las actas de matrimonios. En cualquier caso, los datos sugieren que la emigración es más intensa conforme nos alejamos de la población de referencia (Cáceres en este caso), que se concentran en mayor medida en los territorios ordenados en torno a la red de cañadas procedente de León y, de forma secundaria, en las zonas de origen de los rebaños riojanos y sorianos.

Así pues, las cañadas organizaron las migraciones conectando territorios situados en los extremos de sus trazados a lo largo de un itinerario, vinculando con las zonas de destino un rosario de poblaciones organizadas en torno a ellas. Cabe

${ }^{37}$ Archivo Diocesano de Coria-Cáceres. Libros Parroquiales. Cáceres. Parroquia de San Juan. Los datos que sustentan este comentario se basan en el conteo de contrayentes varones solteros. 
preguntarse, para retomar el hilo de las cuestiones que nos planteábamos anteriormente, si esta estructura organizativa pervive una vez la actividad trashumante ha languidecido presa de la crisis que la paralizó y acabó por desarmarla a partir de 1820 .

Cuadro IV. Procedencias de los contrayentes varones solteros en San Juan de los Borregueros (1750-1850)

\begin{tabular}{cccccc}
\hline $\begin{array}{c}\text { Provincia } \\
\text { Actual }\end{array}$ & Contrayentes & $\begin{array}{c}\text { Provincia } \\
\text { Actual }\end{array}$ & Contrayentes & Provincia \\
Actual & Contrayentes \\
\hline Orense & 6 & Santander & 1 & Soria & 8 \\
Lugo & 3 & Asturias & 6 & Burgos & 2 \\
& & León & 29 & Logroño & 10 \\
& & Zamora & 11 & Palencia & 4 \\
& & Salamanca & 19 & Valladolid & 4 \\
& & Avila & 9 & Madrid & 8 \\
& & & Toledo & 6 \\
\hline
\end{tabular}

\section{CRISTALIZACIÓN DE LAS CORRIENTES MIGRATORIAS PENDULA- RES.}

Desde mediados del siglo XVIII la presencia de estos emigrantes comienza a ser muy visible pero su participación en el tejido social extremeño es constatable ya con frecuencia en el siglo XVI, aunque se trata mayoritariamente de casos aparentemente aislados. Sin embargo, sus lugares de origen se repiten a lo largo de los siglos con constante regularidad. Tal como se indicaba anteriormente, se trata de poblaciones de las montañas de León, de la tierra de Astorga y León, en especial el valle del Esla, lo que nos conecta con los valles de referencia en donde se inicia la cañada zamorana, la llamada cañada de la Plata, la Cañada real leonesa oriental y la occidental; hacia el este, las sierras de Cameros y en la misma latitud, más hacia el este aún, las estivaciones orientales de la sierra de la Demanda, que es el origen de los rebaños sorianos y burgaleses; la sierra de Gredos y la Tierra de Campos, paso obligado de todos ellos.

En los umbrales de la época contemporánea el comportamiento de la emigración con destino en Cáceres fue muy similar al observado durante el conjunto de los tres siglos modernos. Las mismas características básicas se observan sin solución de continuidad. Sin embargo, un análisis más detallado nos permite apuntar ciertos matices que no carecen de importancia. 
Mapa 3a. Procedencias observadas en los matrimonios celebrados en Cáceres durante el siglo XVI

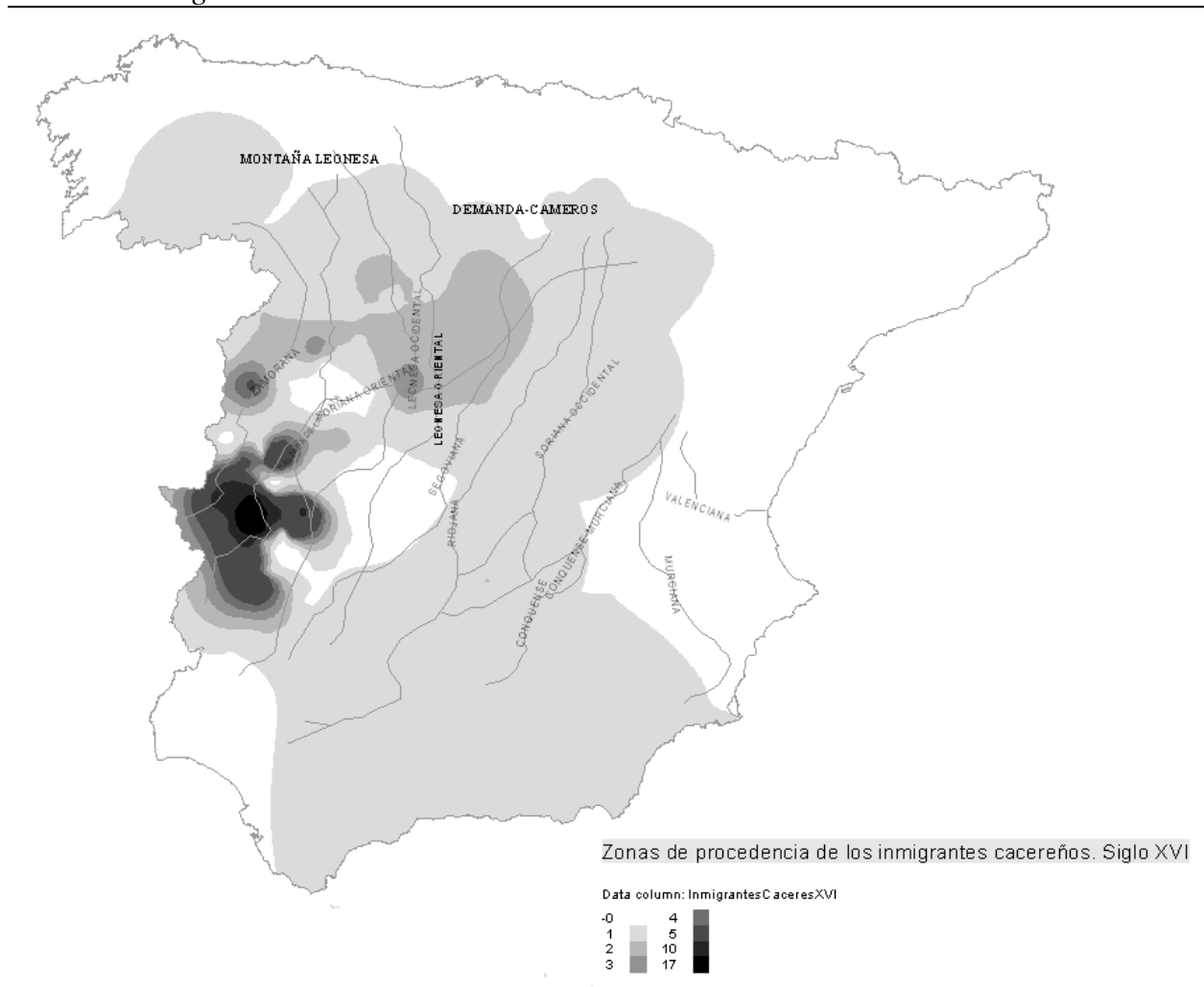

En general, las corrientes migratorias tienden a ampliarse conforme la ciudad gana un papel administrativo del que carece durante la mayor parte de la Época Moderna. Por comparación con la representación anterior, el mapa 3.c. nos muestra que la intensificación de las corrientes migratorias está plenamente integrada en las fuerza de atracción que las nuevas condiciones del mercado de trabajo local ejercían sobre la población joven de las poblaciones vecinas, ampliando la distancia media recorrida por los emigrados considerada globalmente. Creo que es muy significativo que las zonas orientales y meridionales del reino ganen protagonismo, en especial la mancha albacetense, aunque no dejan de ser significativa la continua llegada de Catalanes, aragoneses y, en especial, andaluces. Sin embargo, la importancia cuantitativa que mantienen los territorios septentrionales de procedencia tradicional en Cáceres no sólo aumentan en número, 
sino que cristalizan por primera vez en asentamientos definitivos. Demuestra este hecho, en primera instancia un hecho no incorporado a estos mapas: la incorporación de mujeres al proceso migratorio, lo cual sugiere que los desplazamientos no sólo se realizan de forma estratégicamente planificada, sino que además tiene vocación de estabilidad.

Mapa 3b. Procedencias observadas en los matrimonios celebrados en Cáceres (San Juan) durante el siglo XVIII

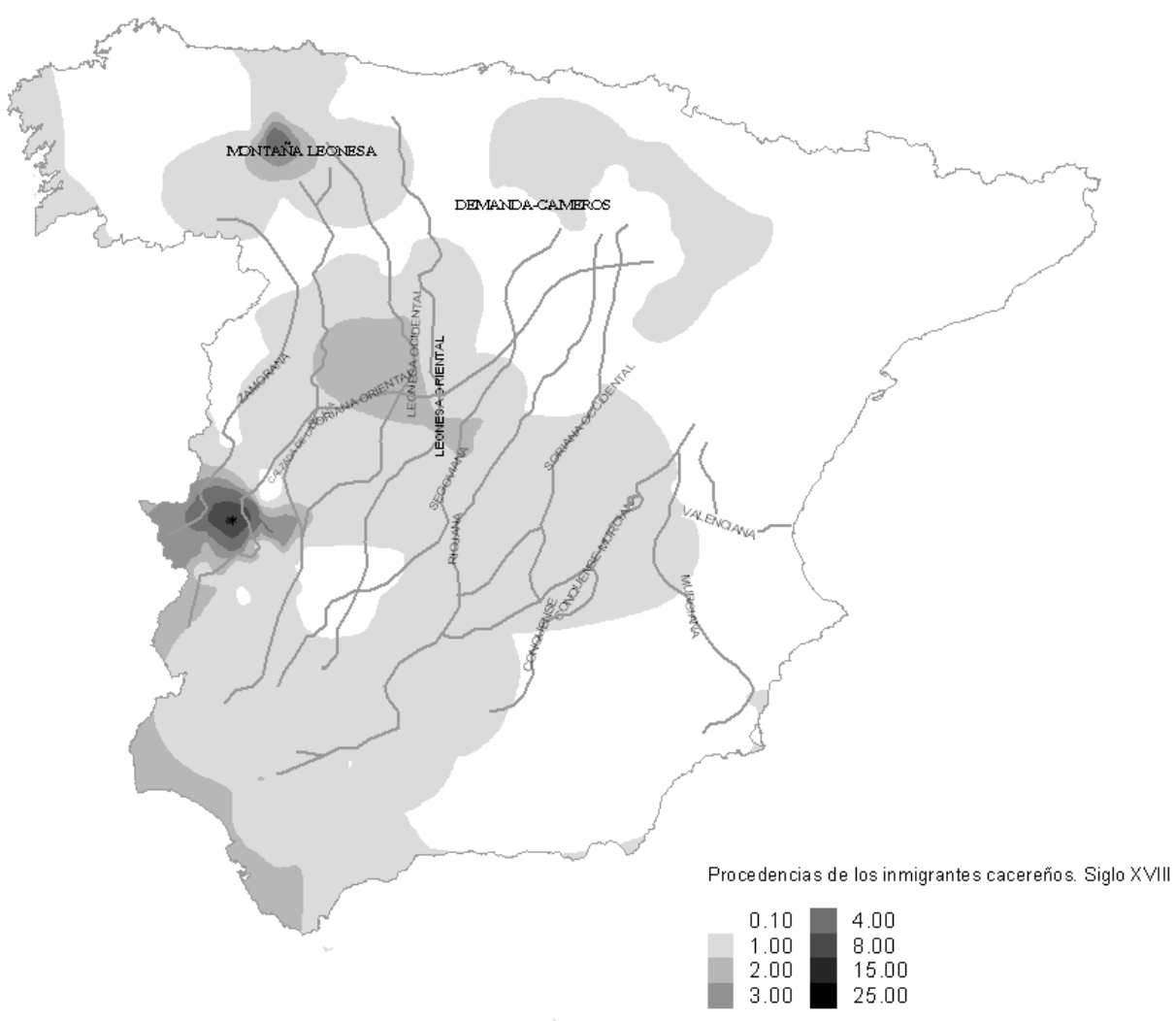

El proceso está documentado por otras vías. Tanto nosotros mismos en varias ocasiones, como otros historiadores -Alfonso Rodríguez, Miguel Ángel Melón y 
últimamente Mercedes Santillana y María de los Ángeles Hernández ${ }^{38}$ - hemos podido constatar que la mecánica de instalación definitiva de las comunidades de cameranos y leoneses, así como la de otras comunidades de emigrantes como los catalanes y aragoneses, sigue una pauta enteramente condicionada por el funcionamiento de las redes parentelares. Esto significa que al establecimiento de los primeros emigrados les sigue la llegada de nuevos integrantes de la familia extensa procedentes de sus comunidades de origen. Los casos son innumerables, y algunos de ellos resultan muy conocidos: la familia García Carrasco, los Viniegra, los Muñoz de San Pedro, los Solo de Zaldívar remiten esencialmente a familias de ganaderos cuyas raíces se encuentra hondamente asentadas en localidades situadas en la Sierra de Cameros. Los Segura, los Calaff, procedentes de Cataluña...

A su alrededor, generaciones sucesivas fueron atrayendo a nuevos emigrantes y tras un proceso de adaptación que conllevaba con frecuencia el matrimonio de estos emigrantes con mujeres pertenecientes a la élite local -hecho similar ocurre en otras poblaciones, como Brozas, por ejemplo, pero también en las grandes villas de la Serena y Tierra de Barros-, lo cual les proporcionaba una posición muy relevante en la comunidad de destino, sus estrategias tendieron siempre a conservar y mejorar, en la distancia, su posición social en las comarcas originarias, en parte por la preservación de lazos que todavía resultaban útiles, en parte por las necesidades de capitales que poco a poco fueron siendo más perentorias, y en parte, finalmente, por la inercia de los grupos sociales a los que pertenecían.

El caso de Antonio Vicente Velázquez es bastante común y nos puede ayudar a comprender este fenómeno de integración social. En este caso, el joven procede de Brieva y entronca con José Blasco, oriundo de Ventrosa, en 1792, el mayor propietario de la colación de San Juan y el principal polo de atracción local de los cameranos de la primera generación, con un inmenso patrimonio construido a partir de la administración de bienes eclesiásticos y nobiliarios, el comercio de lana y la explotación de una cabaña estante y trashumante de enormes dimensiones ${ }^{39}$. En 1802, viudo de su primera mujer, se casó con otra hija de José Blasco, Maria Manuela, natural de Ventrosa, con quien le unía un primer grado de afinidad convenientemente dispensado por el beneficiado Tomás Muñoz Montenegro, que

38 Melón JimÉneZ, M.A. (1999) Antecedentes... op. cit. ; HernándeZ Bermejo M.A y SAntillana Pérez, M. (2009) «Ámbitos de vida cotidiana y espacios vitales de los cacereños que vinieron de lejos (ss. XVIII-XIX)» Original mecanografiado. De próxima aparición en En Norba, Revista de Historia. 24.

${ }^{39}$ El análisis de los libros de Cilla de la parroquia de San Juan (ADC-C, Cáceres, parroquiales, San Juan. Libro de Cilla, $\mathrm{n}^{\circ}$ 55), lo sitúa como el principal contribuyente desde 1784 a 1800 y principal cliente en la venta de lana blanca diezmada por la parroquia. 
como pariente de la familia, había viajado desde Viniegra, de donde procede la rama materna del contrayente, para oficiar la ceremonia.

Mapa 3c. Procedencias observadas en los matrimonios celebrados en Cáceres (San Juan) durante la primera mitad del siglo XIX

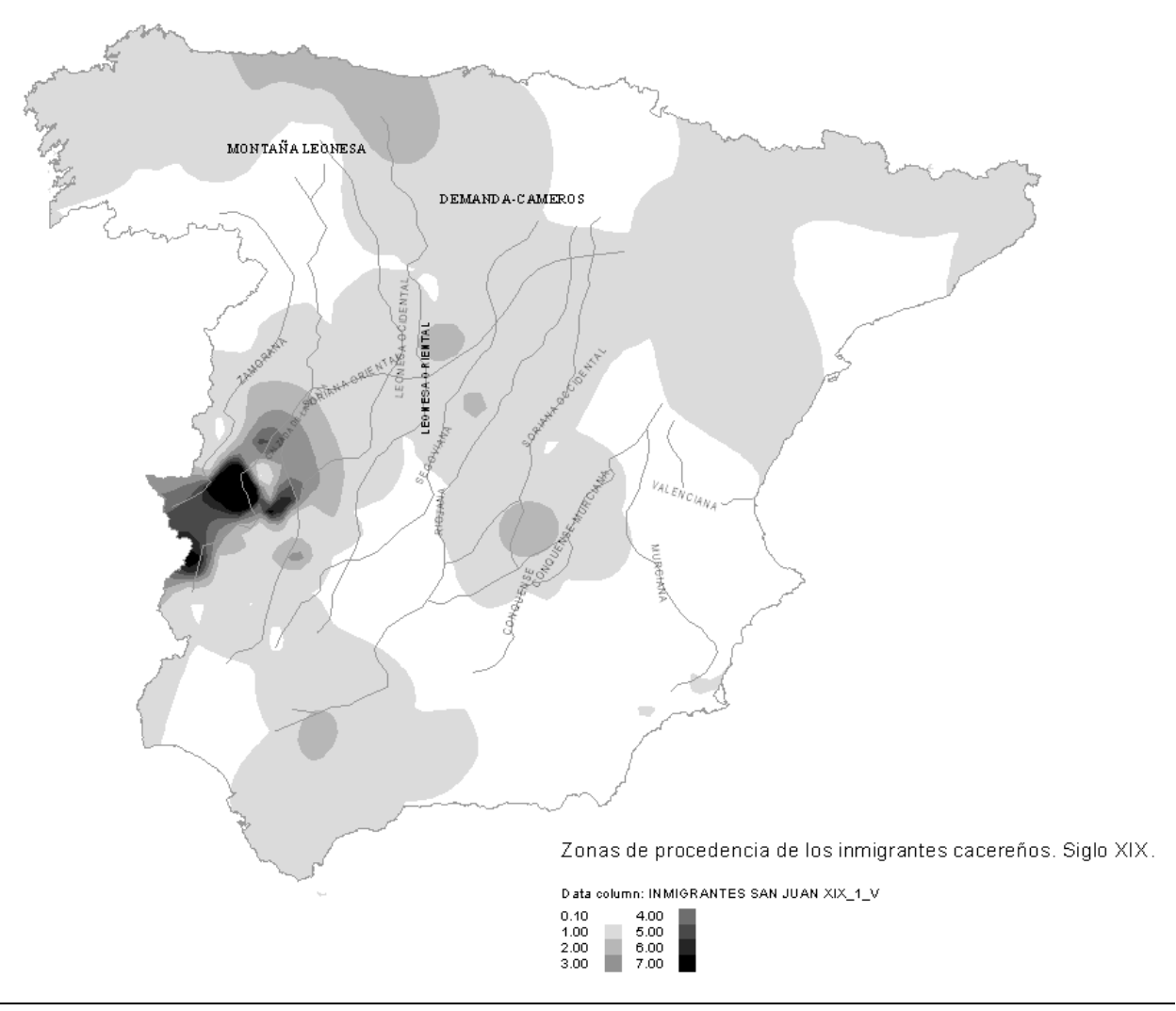

La misma estrategia parece seguirse con la tercera de las hijas de José Blasco Laguna, María Loreto Josefa, que se casó con Andrés Muñoz Montenegro, natural como sus padres de Mansilla de la Sierra, una de las localidades próximas a Los Cameros en la sierra de La Demanda.

Otro ejemplo bien conocido es el de Tomás Muñoz de San Pedro, natural como su hermano Andrés de Mansilla, quien contrajo matrimonio en 1809 con María del Carmen Lizaur y Meneses, hija de Manuel Flores Lizaur, oriundo de Valencia de Alcántara al parecer, e Ignacia Meneses Cabrera, integrante de una de las 
principales familias de hacendados locales que habían probado fortuna con el comercio. Su posición en el elitista grupo de los terratenientes queda bien reflejada en sus extensas redes familiares.

Es obvio que la intención de sendas familias en ambos casos era perpetuar la estrategia de fusión de patrimonios en uno y otro lado extremo de la cañada, hecho que demuestran otros ejemplos de estrategias patrimoniales bien conocidos a través de los estudios de Santillana y Hernández ${ }^{40}$.

En resumen, pues, existe un protagonismo evidente de las cañadas en la canalización de los movimientos migratorios que están presentes en las zonas meridionales de la corona de Castilla durante toda su historia, en especial en los territorios de la antigua provincia de Extremadura. Este papel es aún más significativo por la relevancia que llegaron a alcanzar los trashumantes en el desarrollo del capitalismo primitivo en las grandes villas regionales.

${ }^{40}$ Hernandez Bermejo, M.A. y SAntillana Pérez, M. (2010) «Ámbitos...» op. cit. 